required to answer SF36 questionnaire at baseline to determine their HRQOL status after accident. However only those in the intervention group were given coaching, which was delivered concurrent to usual care. The effectiveness of this intervention was measured after all respondents in both groups had finished intervention and usual care respectively-they were required to answer SF36 questionnaire once again.

Results The follow up stage is still ongoing. However, the result will be ready at the time of this conference. Potential results are the mean of HRQOL will be reduced more significantly among those in the intervention group compare to those who are only receive usual care. Low self-esteem among these survivors will be improved as this intervention provides support to regain self-confidence that has lost due to the injury.

Conclusions We are absolutely convinced that the module in this intervention is able to assist injured person to improve their emotional wellbeing. Therefore the combination of biopsychosocial coaching intervention with usual care is believed to give synergistic effect to the positive changes on HRQOL status.

\section{INCIDENCE, COSTS AND OUTCOMES OF NON-UNION, DELAYED UNION AND MAL-UNION FOLLOWING LONG BONE FRACTURE}

${ }^{1}$ Christina L Ekegren, ${ }^{1}$ Belinda J Gabbe, ${ }^{1,2}$ Elton R Edwards, ${ }^{3,4}$ Richard de Steiger, ${ }^{5,6}$ Richard Page. ${ }^{1}$ Monash University, Australia; ${ }^{2}$ The Alfred Hospital, Australia; ${ }^{3}$ Epworth Healthcare, Australia; ${ }^{4}$ University of Melbourne, Australia; ${ }^{5}$ University Hospital Geelong, Australia; ${ }^{6}$ Deakin University, Australia

\subsection{6/injuryprev-2016-042156.791}

Background The management of long bone fractures is complex and the risk of healing complications persists. Previous studies have reported widely varying incidence and cost estimates for fracture healing complications, with the majority focusing on small cohorts with specific fracture types and treatment methods. Also, there is a lack of research on patient outcomes. The aim of this study was to describe the incidence, inpatient costs and 12month outcomes of readmissions for healing complications following long bone fracture.

Methods All humeral, tibial and femoral fractures registered by the Victorian Orthopaedic Trauma Outcomes Registry over a 5-year period were linked with hospital data to identify 2-year readmissions for non-union, delayed union or mal-union. Study outcomes for patients with complication readmissions included hospital length of stay, inpatient costs, function (Extended Glasgow Outcome Scale), work status and quality of life (EQ-5D) measured 12 months post-injury. Cases with and without complications were compared using univariable and multivariable methods.

Results Of the 3908 patients included, 9\% were readmitted for healing complications within 2 years of their index fracture. The most common complication type was non-union (77\% of complications). Admissions for fracture healing complications incurred an extra 3 days in hospital and costs of up to AUD $\$ 25,000$ per patient (AUD \$5.4 M in total). Patients with healing complications reported worse function, quality of life and return to work rates 12 months post-injury. After adjusting for key confounders, patients had higher odds of developing complications if they were older, receiving compensation or had multiple fractures.

Conclusions Patients who develop complications have poorer outcomes and place additional burden on healthcare and compensation systems. As such, there is a need to prioritise future research aimed at preventing fracture healing complications and improving patient outcomes.

\section{MANAGEMENT OF STRESS \& MUSCULOSKELETAL DISORDERS IN THE WORKPLACE BY YOGA, MEDITATION AND THERAPEUTIC REFLEXOLOGY}

'PK Goswami, ' 2 Samantha Jane Burnell. 'Labour Department, Government of Delhi; ${ }^{2} T$ The International Academy of Reflexology and Meridian Therapy, South Africa

\subsection{6/injuryprev-2016-042156.792}

Problem $\mathrm{n}$ the modern work environments of today, we have started putting strain on our systems as a whole- due to physical, mental and emotional demands far surpassing what our systems can bear. These exposures are going against the natural laws of nature and often done without conscious awareness of the longer-term damage. There is an extended list of occupational disorders that exist, nowadays, due to the upright skeletal position of the man, and more so because of the uninvited pressures that he has put onto the skeletal structure as a whole. The result equals the vertebral column being unable to withstand the stresses of prolonged standing or sitting down, especially when unsupported.

Description of problem Musculoskeletal disorders and diseases are common occupational problems all over the world. They are a result of the previous research of author "Health hazards in Small and Medium Enterprises garment industries in developing countries" has been taken for this further study. The previous study was conducted in Delhi from August-2014 to December2014. The database and result of the study with respect to Indian population has been chosen for this further study. The study revealed that musculoskeletal problems (54\%) were the commonest health problem in India. The objective of the study is to design an effective program to create an awareness surrounding the management of stress, Musculoskeletal problems as well as an effective management of pain related symptoms by using Yoga, meditation and Therapeutic Reflexology and Meridian Therapy.

Result After an intervention by Yoga and Meditation therapies for prevention and cure of Musculoskeletal problems, as well as Reflexology treatments for an on-going maintenance of immediate pain relief, it is found that $27 \%$ workers suffering from acute musculoskeletal problem and $83 \%$ workers suffering from moderate Musculoskeletal problems are no longer experiencing chronic symptoms or completely cured of pain. The Study period is January 2015 to July 2015.

Conclusion Yoga and Meditation is a philosophy and practice that connects the body, breath, and mind to energise and balance the whole system in our body and Reflexology works on the premise that disease is caused by blockages along the meridians, and therefore the treatment is conducted to increase the circulation within the body, clear congestions along the pathways and stimulate the body's own healing potential. Yoga and Meditation can be used for managing Musculoskeletal problems and furthermore, there is vast scope of research for inclusion of Reflexology and Meridian therapy sessions for immediate and maintained pain relief results in this program.

\section{ANALYSIS OF THE EMERGENCY MEDICAL SERVICES' RESPONSE TO ROAD TRAFFIC INJURIES IN MEXICO}

'Juan Daniel Vera-López, ${ }^{1}$ Ricardo Pérez-Núñez, ${ }^{2}$ Lourdes García-Gómez, ${ }^{2}$ Elisa HidalgoSolozano. 'Secretariado Técnico De Consejo Nacional Para La Prevención De Accidentes, México D.F; ${ }^{2}$ National Institute of Public Health of Mexico

10.1136/injuryprev-2016-042156.793 